\title{
Effects of Japanese-style online hemodiafiltration on survival and cardiovascular events
}

Kazuyoshi Okada ${ }^{1 *}$, Hiroyuki Michiwaki ${ }^{2}$, Manabu Tashiro ${ }^{1}$, Tomoko Inoue ${ }^{1}$, Hisato Shima ${ }^{1}$, Jun Minakuchi ${ }^{1}$ and Shu Kawashima'

\begin{abstract}
Background: All-cause mortality can be reduced in patients receiving European-style high-volume post-dilution online hemodiafiltration (post-OHDF) compared with hemodialysis (HD). Japanese-style high-volume pre-dilution online hemodiafiltration (pre-OHDF) can also decrease all-cause mortality. No studies have investigated survival in patients receiving Japanese-style post-OHDF, and directly compared survival between pre-OHDF and post-OHDF. This study sought to clarify these issues.

Methods: We conducted this retrospective observational study at multiple facilities of our medical corporation, which have similar quality of healthcare management, from July 1, 2017 to July 1, 2020. Japanese-style OHDF included pre-OHDF and post-OHDF. Pre-OHDF and post-OHDF were each divided into high-volume and low-volume subgroups. We compared 3-year all-cause mortality and cardiovascular events between OHDF and high-flux HD using propensity score matching. In addition, we examined the effects of different modalities through comparisons between high-volume and low-volume pre-OHDF and between pre-OHDF and post-OHDF.

Results: Post-OHDF was associated with significantly lower all-cause mortality $(P=0.004$, log-rank test; hazard ratio [HR] 0.257, 95\% confidence interval [Cl] 0.094-0.701) and cardiovascular events during all 3 years $(P<0.05)$ compared with HD. High-volume post-OHDF was associated with lower all-cause mortality compared with hemodialysis (logrank test $P=0.022$ ) but low-volume post-OHDF was not. Pre-OHDF was also associated with lower all-cause mortality $(P<0.001$, log-rank test; HR 0.316; 95\% Cl 0.212-0.472) compared with HD but was not associated with cardiovascular events. All-cause mortality did not significantly differ between post-OHDF and pre-OHDF.
\end{abstract}

Conclusions: These findings suggest that Japanese-style post-OHDF may improve all-cause mortality to a level similar to that of pre-OHDF and that post-OHDF, particularly high-volume post-OHDF may reduce cardiovascular events.

Keywords: Post-dilution online hemodiafiltration, Pre-dilution online hemodiafiltration, Survival, Cardiovascular events

*Correspondence: kokada@khg.or.jp

${ }^{1}$ Department of Nephrology, Kawashima Hospital, 6-1 Kitasakoichiban-cho, Tokushima-shi, Tokushima 770-0011, Japan

Full list of author information is available at the end of the article

\section{Background}

Online hemodiafiltration (OHDF) has two different modes, pre-dilution OHDF (pre-OHDF) and postdilution OHDF (post-OHDF), depending on where the substitution fluid is administered in the extracorporeal circuit. The dialysate flow rate (QD) in OHDF is reduced because part of the dialysate is used as the original author(s) and the source, provide a link to the Creative Commons licence, and indicate if changes were made. The images or other third party material in this article are included in the article's Creative Commons licence, unless indicated otherwise in a credit line to the material. If material is not included in the article's Creative Commons licence and your intended use is not permitted by statutory regulation or exceeds the permitted use, you will need to obtain permission directly from the copyright holder. To view a copy of this licence, visit http://creativecommons.org/licenses/by/4.0/. The Creative Commons Public Domain Dedication waiver (http://creativecommons.org/publicdomain/zero/1.0/) applies to the data made available in this article, unless otherwise stated in a credit line to the data. 
substitution fluid. The convection volume $(\mathrm{CV})$ is the sum of the substitution volume and ultrafiltration volume. The removal characteristics of solutes differ according to the dilution method used and the performance of the hemodiafilter. In post-OHDF in Europe, the $\mathrm{CV}$ is approximately $16-26 \mathrm{~L}(66.7-108.3 \mathrm{~mL} /$ min) with low-permeability hemodiafilters, and substitution volumes greater than $20 \mathrm{~L}$ require increasing the blood flow rate $(\mathrm{QB})$ to greater than $380 \mathrm{~mL} /$ min in order to control the transmembrane pressure and filtration fraction [1]. Because QB of $300 \mathrm{~mL} / \mathrm{min}$ or higher is rarely obtained in Japan, Japanese-style OHDF has become widely used. In Japanese-style preOHDF and post-OHDF, the substitution volumes are approximately $24-84 \mathrm{~L}(100-350 \mathrm{~mL} / \mathrm{min})$ and $6-16$ $\mathrm{L}$ (25.0-66.7 $\mathrm{mL} / \mathrm{min})$, respectively, with moderate- to high- permeability of the hemodiafilter. Pre-OHDF has been selected over post-OHDF to prevent clogging of the hemodiafilter and excessive albumin leakage due to hemoconcentration induced by the low QB. Given that the movement of lower molecular weight solutes depends mainly on diffusion, $\mathrm{Kt} / \mathrm{V}$ decreases in preOHDF with a reduction of QD due to the large substitution volume. Therefore, some facilities increase the total QD (QD including substitution volume) from 500 to $600-700 \mathrm{~mL} / \mathrm{min}$ to compensate for the decrease in QD or keep the total QD constant at $500 \mathrm{~mL} / \mathrm{min}$ to stabilize the environment in the hemodiafilter as in hemodialysis (HD), depending on the facility policy. On the other hand, the difference in substitution volume in post-OHDF between Japan and Europe is mainly due to the permeability of the hemodiafilter.

The purpose of OHDF and HD is to improve clinical symptoms and extend patient survival by removal of uremic toxins. Hemodiafilters and dialyzers that are permeable to solutes have been developed in order to remove a large amount of uremic toxins. A high-flux dialyzer can remove uremic toxins with a molecular weight cutoff of $25 \mathrm{kDa}$. The ability to remove larger uremic toxins depends primarily on the convection and substitution volumes. Therefore, OHDF has been developed mainly for removal of a wide range of middle to high molecular weight solutes that are not sufficiently removed by HD. Given the evidence showing that $\beta_{2}$-microglobulin $\left(\beta_{2} \mathrm{MG}\right)$ is a uremic toxin, it is now routine practice to increase the removal of $\beta_{2} \mathrm{MG}$, which has a molecular weight of $11.8 \mathrm{kDa}$, and some cytokines by post-OHDF. However, sufficient removal of high molecular weight solutes using low-permeability hemodiafilters requires a considerable increase in the substitution volume (defined as European-style post-OHDF). Japanese-style OHDF has been also performed using low-permeability hemodiafilters in cases of hypoalbuminemia.
The number of patients treated by Japanese-style postOHDF has been too small to study using the Japanese Society for Dialysis Therapy Renal Data Registry (JRDR) database with propensity score matching (PSM). Our medical corporation owns multiple facilities at which 1,010 patients received maintenance dialysis in 2021 (HD 40.6\%, pre-OHDF 39.7\%, post-OHDF $18.9 \%$, intermittent infusion hemodiafiltration $0.8 \%$ ). These facilities were considered suitable for investigating Japanese-style OHDF because they all provide similar healthcare quality and use similar dialysis conditions, including the same total QD of $500 \mathrm{~mL} / \mathrm{min}$, and there seems to be no significant between-center difference in unobserved background factors. This study compared all-cause mortality between Japanese-style post-OHDF, pre-OHDF and HD, examined whether pre-OHDF would provide results similar to those reported previously [2], and investigated whether OHDF would decrease the number of hospitalizations and cardiovascular events in comparison with HD.

\section{Methods \\ Ethics}

This study was approved by the Research Ethics Committee of Kawashima Hospital and all clinical investigations were conducted according to the principles expressed in the Declaration of Helsinki. All patients provided informed consent for their data to be included in the study.

\section{Patient selection}

Of the 944 patients undergoing maintenance dialysis with HD or OHDF at our facilities as of July 1, 2017, this study analyzed 797 patients, including 356 who were receiving HD and 441 who were receiving OHDF. The patients treated with OHDF were further divided into a preOHDF group $(n=345)$ and a post-OHDF group $(n=95)$. Then, we compared survival between the HD group and the pre-OHDF and post-OHDF groups using PSM. The pre-OHDF and post-OHDF groups were each further divided into low-volume and high-volume subgroups by the median and by the weighted average. We compared survival between each subgroup and the HD group, and between pre-OHDF and post-OHDF using PSM. The exclusion criteria were age under 20 years, blood purification methods other than HD or OHDF, dialysis frequency of less than three sessions per week, dialysis time less than $3 \mathrm{~h}$, and pregnancy or lactation. We excluded patients whose dialysis conditions (dialysis method, dilution method, substitution volume, and membrane material) at the start of the study on July 1, 2017 were different from their dialysis conditions used on April 1, 2017. Patients receiving HD, pre-OHDF, or post-OHDF 
were defined as those who received the same dialysis method for 3 years (July 1, 2017 to July 1, 2020). Patients who continued the same dialysis conditions during this 3 -year period were included. The modality was chosen at the physician's discretion. The variables related to dialysis conditions to be censored every year differed depending on the combinations analyzed. Furthermore, cases that moved from one group to the other across the median or weighted average were censored every year in comparisons between groups, and such cases were censored daily in the Kaplan-Meier survival curve. The period before the change in dialysis conditions and movement to another group was subjected to statistical analysis. The results of blood tests were extracted from the medical records.

\section{Preparation of propensity score-matched pairs}

To analyze the effects of OHDF and HD on survival outcomes, propensity scores were matched in 336 pairs of patients receiving OHDF or HD. To analyze the effects of pre-OHDF and HD on survival outcomes, propensity scores were matched in 288 pairs of patients receiving pre-OHDF or HD. Propensity scores were matched to compare the effects of low-volume (less than the median or weighted average) and high-volume (at least the median or weighted average) pre-OHDF versus HD on survival outcomes. There were 144 matched patients receiving low-volume pre-OHDF and 190 matched patients receiving high-volume pre-OHDF by both the median and the weighted average. Propensity scores were also matched in 88 pairs of patients receiving postOHDF or HD to analyze the effects of post-OHDF and HD on survival outcomes. To compare the effects of low-volume and high-volume post-OHDF vs. HD on survival outcomes, propensity scores were matched in 23 matched patients receiving low-volume post-OHDF and 65 matched patients receiving high-volume post-OHDF by the median and in 64 matched patients receiving lowvolume post-OHDF and 27 matched patients receiving high-volume post-OHDF by the weighted average.

Propensity scores were also matched to compare the direct effects of low-volume and high-volume pre-OHDF on survival outcomes. The number of pairs was the same $(n=144)$ in both groups according to the median and weighted average. Moreover, low-volume and high-volume post-OHDF and low-CV and high-CV post-OHDF according to the median (25 pairs and 28 pairs) and those according to the weighted average (27 pairs and 28 pairs) were also compared. In addition, propensity scores were matched in 96 pairs of patients receiving pre-OHDF or post-OHDF to analyze the effects of pre-OHDF and postOHDF on survival outcomes.
The incidence of hospitalization and cardiovascular events in the HD, OHDF, pre-OHDF, and post-OHDF groups were evaluated according to total number of occurrences per year. A cardiovascular event was defined as any of the following conditions requiring intervention or surgery regardless of whether hospitalization was required or treatment was provided on an outpatient basis: angina/myocardial infarction, arteriosclerosis obliterans/peripheral arterial disease, or stenosis/occlusion of vascular access.

PSM was performed for 27 items, as in the previous study that confirmed the effects of pre-OHDF [7]. If the number of cases was less than 30, matching was performed with nine variables. Missing variables were filled with the average value. If the percentage of missing values was $10 \%$ or more of all cases, that variable was excluded from PSM.

To calculate the propensity score for each patient, multivariable logistic regression analysis was performed using the treatment group as a dependent variable and the covariates as independent variables, followed by logit transformation. The propensity scores were calculated to a precision of 14 decimal places. Regardless of whether the number of cases was large or not, patients in the two groups were paired by nearest available matching with a caliper (0.23456) of $0.2 \times \mathrm{SD}$ of logit values for all patients in both groups [3].

\section{Survival analysis, cardiovascular events analysis, and statistics}

Survival and total number of cardiovascular events requiring intervention or surgery per year were analyzed using the medical records, which included information on deaths, hospitalizations, discharges, transfers to other hospitals, and details of operations and interventions. A daily survival analysis was performed for the two groups with censored cases using the Kaplan-Meier method. The statistical significance of differences between groups was determined using the log-rank test. Cox proportional hazard model was used to calculate the hazard ratio between treatment groups, and to evaluate the relationship between mortality and patients' backgrounds. Mann-Whitney U-test was used to compare the total number of hospitalization and cardiovascular events, and multiple regression analysis was used to evaluate the relationship between annual hospitalizations or cardiovascular events and patients' backgrounds in each treatment group.

All analyses were performed using SPSS Statistics for Windows, version 25 (IBM Corporation, Armonk, NY). A $P$-value $<0.05$ (two-tailed) was considered statistically significant. 


\section{Results}

\section{Patient selection}

In total, 944 patients were selected from the medical records held by our corporation according to the flow diagram shown in Fig. 1. A total of 797 patients, including 356 who were receiving $\mathrm{HD}$ and 441 who were receiving OHDF (pre-OHDF, $n=345$; post-OHDF, $n=96$ ) were included in the propensity score-matched model.

\section{Comparison of patient characteristics before and after PSM}

The variables in the HD, OHDF, pre-OHDF, and postOHDF groups before PSM are shown in Table 1. Because the ferritin- value was missing at the start of the study for 1 case, the average ferritin value for the same case in 2018, 2019, and 2020 was substituted.

The variables before and after PSM in the OHDF, preOHDF, and post-OHDF groups versus HD, and those in the pre-OHDF group vs. the post-OHDF group are shown in Additional files 1-10: Tables S1-S10.

\section{Comparison of patient survival in the OHDF, pre-OHDF, and post-OHDF groups versus the HD group}

Three-year all-cause mortality was significantly lower in the OHDF group than in the HD group $(P<0.001$, log-rank test; hazard ratio [HR] $0.276 ; 95 \%$ confidence interval [CI] 0.189-0.404; Additional file 11: Figure S1). When the caliper value was reduced to 0.001 , all significant differences in background factors disappeared but the significant difference in all-cause mortality remained $(P=0.001$, log-rank test). Three-year all-cause mortality was significantly lower in the pre-OHDF group than in the HD group $(P<0.001$, log-rank test; HR 0.316; $95 \%$ CI $0.212-0.472$; Fig. 2a) and the difference of mortality between pre-OHDF and HD was $26 \%$ in 3 years.

When the two groups were divided by the median (72 L), all-cause mortality was significantly lower in both the high-volume $(80.4 \pm 5.5 \mathrm{~L})$ and low-volume $(58.7 \pm 5.1 \mathrm{~L})$ pre-OHDF groups compared with the HD group (highvolume pre-OHDF: $P<0.001$, log-rank test; HR 0.418, 95\% CI 0.254-0.690; Fig. 2b; low-volume pre-OHDF: $P<0.001$, log-rank test; HR 0.264; 95\% CI 0.138-0.507; Fig. 2c). The result was the same when the two groups were divided by the weighted average $(71.3 \mathrm{~L})$.

Three-year all-cause mortality was significantly lower in the post-OHDF group than in the HD group $(P=0.004$, log-rank test; HR 0.257; 95\% CI 0.094-0.701; Fig. 3a). Although the median (10 L) was not significantly different in the high-volume $\geq 10 \mathrm{~L}(11.2 \pm 1.8 \mathrm{~L})$ postOHDF group ( $P=0.051$; Fig. $3 \mathrm{~b})$, the weighted average (10.4 L) was significantly lower in the high-volume $\geq 12$

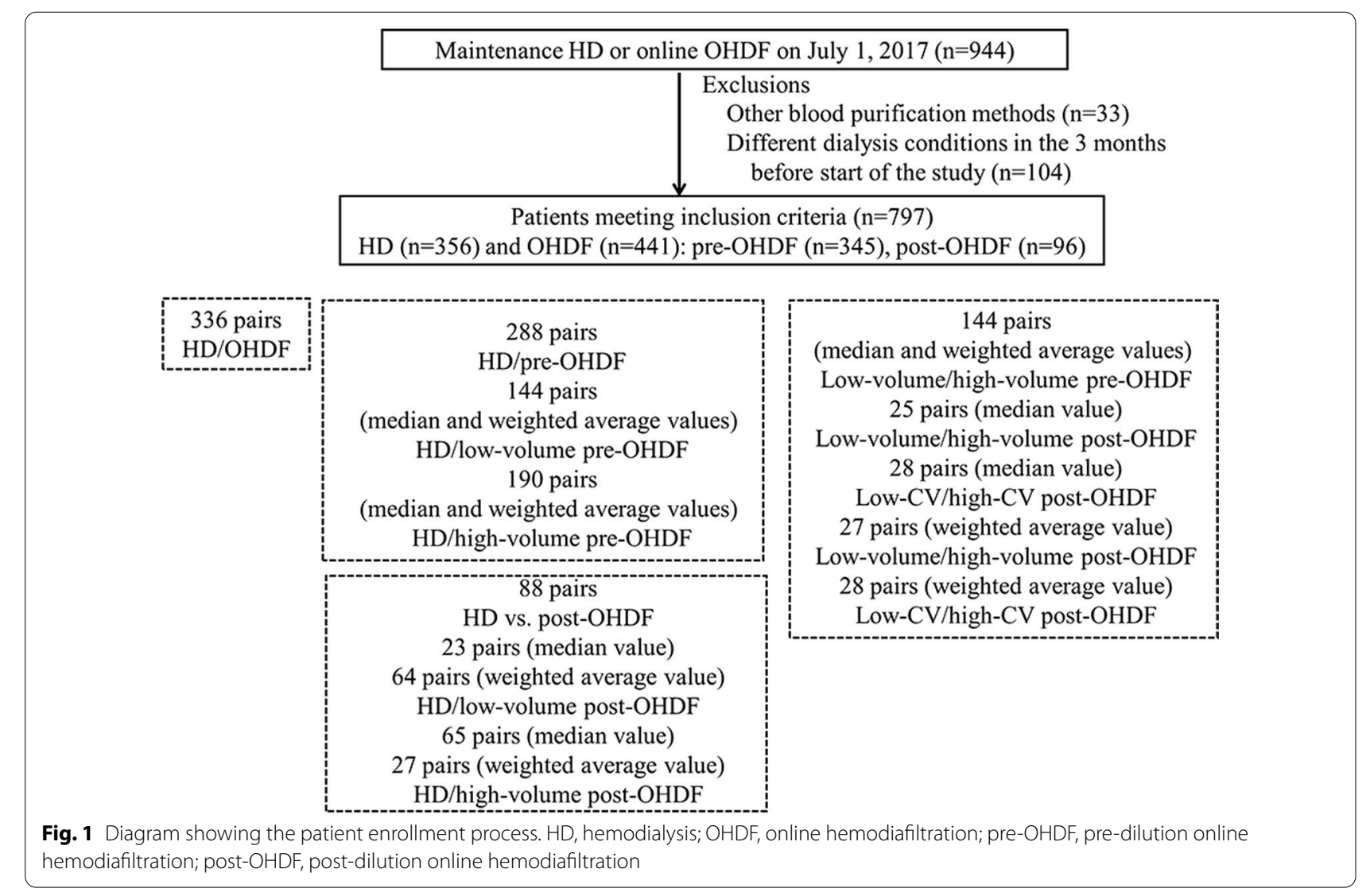


Table 1 Background factors in the HD, OHDF, pre-OHDF, and post-OHDF groups

\begin{tabular}{|c|c|c|c|c|c|c|}
\hline Item & HD & OHDF & $p$-value ${ }^{1)}$ & pre-OHDF & post-OHDF & $p$-value ${ }^{2)}$ \\
\hline Total $(n)$ & 356 & 441 & & 345 & 96 & \\
\hline Sex, male/female (\%) & $64.0 / 36.0$ & $66.4 / 33.6$ & 0.528 & $66.1 / 33.9$ & $67.7 / 32.3$ & 0.746 \\
\hline Underlying disease & & & $<0.001$ & & & $<0.001$ \\
\hline Chronic nephropathy (\%) & 26.7 & 39.5 & & 36.5 & 50.0 & \\
\hline Diabetic nephropathy (\%) & 34.4 & 30.8 & & 32.5 & 25.0 & \\
\hline Nephrosclerosis (\%) & 17.4 & 7.9 & & 8.7 & 5.2 & \\
\hline Other (\%) & 21.6 & 21.8 & & 22.3 & 19.8 & \\
\hline Diabetes mellitus, yes/no (\%) & $38.5 / 61.5$ & $33.1 / 66.9$ & 0.133 & $34.5 / 65.5$ & $28.1 / 71.9$ & 0.148 \\
\hline History of myocardial infarction, yes/no (\%) & $1.7 / 98.3$ & 2.0/98.0 & 0.916 & 2.0/98.0 & $2.1 / 97.9$ & 0.943 \\
\hline History of intracerebral bleeding, yes/no (\%) & $0.6 / 99.4$ & $0.9 / 99.1$ & 0.882 & $0.9 / 99.1$ & $1.0 / 99.0$ & 0.842 \\
\hline History of cerebral infarction, yes/no (\%) & $4.8 / 95.2$ & $3.4 / 96.6$ & 0.423 & $3.2 / 96.8$ & $4.2 / 95.8$ & 0.562 \\
\hline History of quadruple amputation, yes/no (\%) & $0.8 / 99.2$ & $1.1 / 98.9$ & 0.958 & $1.2 / 98.8$ & 1.0/99.0 & 0.915 \\
\hline Use of antihypertensive agents, yes/no (\%) & $40.2 / 59.8$ & $37.0 / 63.0$ & 0.394 & $37.4 / 62.6$ & $35.4 / 64.6$ & 0.613 \\
\hline Age (years) & $72.6 \pm 10.9$ & $64.6 \pm 11.7$ & $<0.001$ & $65.4 \pm 11.7$ & $61.8 \pm 11.3$ & $<0.001$ \\
\hline Dialysis vintage (months) & $98.2 \pm 93.1$ & $145.2 \pm 118.4$ & $<0.001$ & $144.8 \pm 118.7$ & $146 \pm 118.0$ & $<0.001$ \\
\hline Blood flow rate (mL/min) & $268.0 \pm 19.8$ & $279.5 \pm 20.3$ & $<0.001$ & $279.0 \pm 19.7$ & $281.3 \pm 22.4$ & $<0.001$ \\
\hline Duration of dialysis session (h) & $4 \pm 0$ & $4 \pm 0$ & $>0.999$ & $4 \pm 0$ & $4 \pm 0$ & $>0.999$ \\
\hline Systolic BP, predialysis (mmHg) & $142 \pm 23$ & $144 \pm 25$ & 0.334 & $143 \pm 26$ & $146 \pm 21$ & 0.461 \\
\hline Diastolic BP, predialysis (mmHg) & $74 \pm 13$ & $77 \pm 14$ & $<0.001$ & $77 \pm 15$ & $79 \pm 13$ & $<0.001$ \\
\hline Body mass index (calculated as kg/m²) & $21.5 \pm 3.8$ & $22.7 \pm 3.8$ & $<0.001$ & $22.7 \pm 3.8$ & $22.8 \pm 4.1$ & $<0.001$ \\
\hline Albumin, predialysis (g/dL) & $3.3 \pm 0.4$ & $3.4 \pm 0.3$ & 0.001 & $3.4 \pm 0.3$ & $3.3 \pm 0.3$ & $<0.001$ \\
\hline High-sensitivity CRP, predialysis (g/dL) & $0.566 \pm 1.377$ & $0.404 \pm 1.168$ & 0.072 & $0.403 \pm 1.122$ & $0.406 \pm 1.326$ & 0.199 \\
\hline Corrected calcium, predialysis (m/dL) & $9.2 \pm 0.7$ & $9.2 \pm 0.7$ & 0.767 & $9.2 \pm 0.7$ & $9.2 \pm 0.6$ & 0.793 \\
\hline Phosphorus, predialysis (m/dL) & $4.9 \pm 1.5$ & $5.5 \pm 1.4$ & 0.000 & $5.5 \pm 1.4$ & $5.5 \pm 1.6$ & $<0.001$ \\
\hline Hemoglobin, predialysis (m/dL) & $11.0 \pm 1.2$ & $11.2 \pm 1.2$ & 0.023 & $11.2 \pm 1.3$ & $11.2 \pm 1.1$ & 0.069 \\
\hline Ferritin (ng/mL) & $75.4 \pm 89.8$ & $72.7 \pm 134.5$ & 0.753 & $75.7 \pm 147.4$ & $61.5 \pm 70.1$ & 0.546 \\
\hline $\mathrm{Kt} / \mathrm{N}$ & $1.7 \pm 0.3$ & $1.6 \pm 0.3$ & $<0.001$ & $1.6 \pm 0.3$ & $1.7 \pm 0.3$ & $<0.001$ \\
\hline Creatinine, predialysis (mg/dL) & $9.39 \pm 3.04$ & $11.34 \pm 2.93$ & $<0.001$ & $11.21 \pm 2.86$ & $11.82 \pm 3.12$ & $<0.001$ \\
\hline Blood urea nitrogen, predialysis (mg/dL) & $54.6 \pm 16.0$ & $58.8 \pm 14.1$ & $<0.001$ & $59.0 \pm 14.3$ & $58.0 \pm 13.1$ & $<0.001$ \\
\hline \multicolumn{7}{|l|}{ Substitution volume (L) } \\
\hline Mean & & & & $71.3 \pm 12.0$ & $10.4 \pm 2.2$ & \\
\hline Median (quartile range) & & & & $72(60-84)$ & $10(8-12)$ & \\
\hline
\end{tabular}

$\mathrm{HD}$, hemodialysis; OHDF, online hemodiafiltration; pre-OHDF predilution online hemodiafiltration; post-OHDF, postdilution online hemodiafiltration; $\mathrm{BP}$, blood pressure; CRP, C-reactive protein

1) Test between HD and OHDF (Chi squared test or t-test); 2) Test among OHDF, pre-OHDF and post-OHDF (Chi squared test or One-way ANOVA)

$\mathrm{L}(13.2 \pm 1.7 \mathrm{~L})$ post-OHDF group than in the HD group $(P=0.022$, log-rank test; Fig. 3c). However, the HR and 95\% CI could not be calculated according to the weighted value because there were no deaths. There was no significant difference in mortality between the low-volume post-OHDF group by the median or weighted average and the HD group.

\section{Comparison of patient survival between the pre-OHDF and post-OHDF groups}

There was no significant difference in any of the variables (Additional file 10: Table S10) or in 3-year all-cause mortality (Fig. 4) between the pre-OHDF and postOHDF groups.

\section{Comparison of patient survival for the high-volume versus low-volume pre-OHDF and post-OHDF groups}

There was no significant difference in any variable between the high-volume and low-volume pre-OHDF groups divided by the median or weighted average (Table 2). There was also no significant difference in 3-year all-cause mortality between the high-volume and low-volume pre-OHDF groups (Fig. 5). Moreover, there was no significant difference in mortality between the high-volume and low-volume post-OHDF groups 


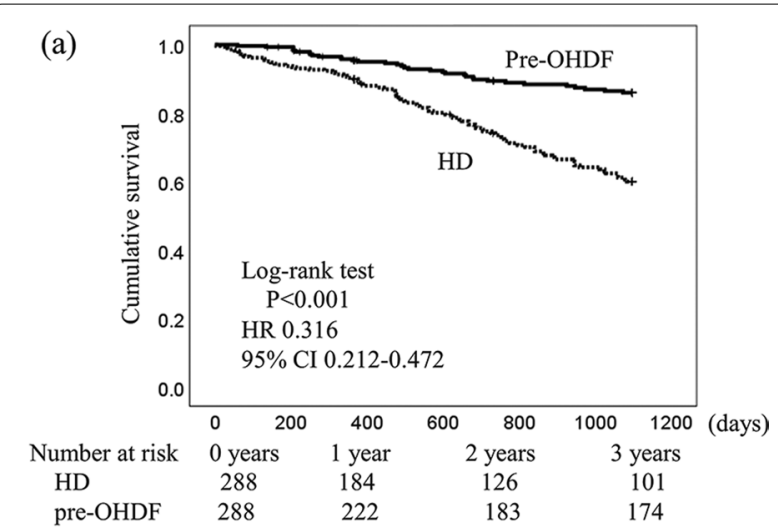

(b)

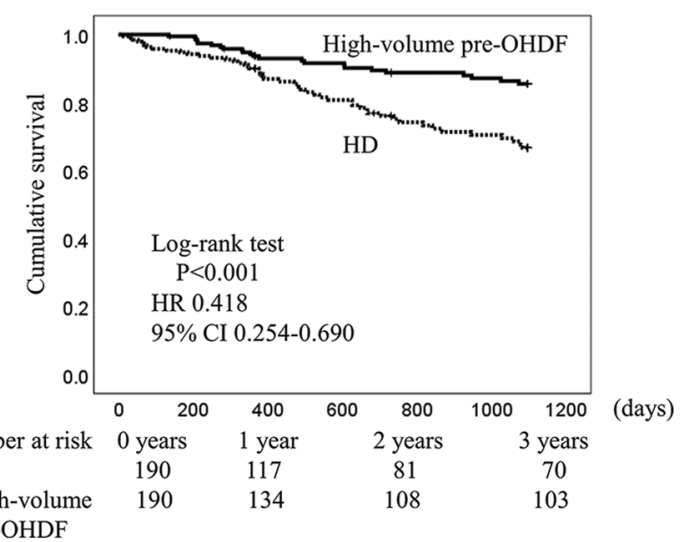

(c)

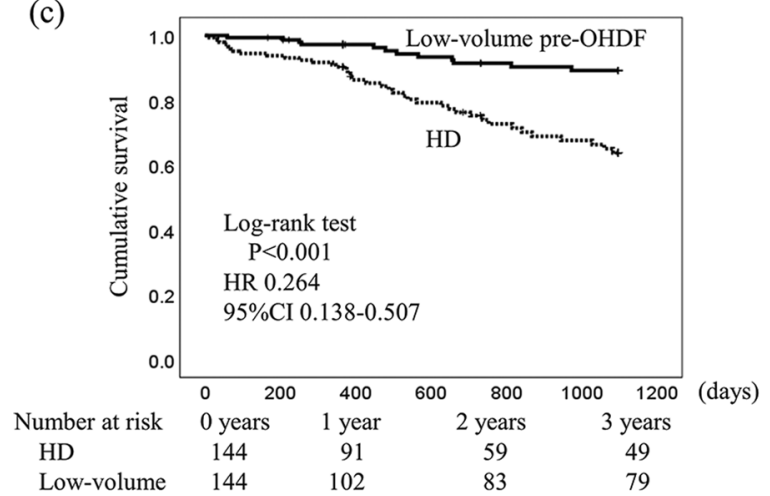

Low-volume pre-OHDF

Fig. 2 Comparison of patient survival outcomes in the pre-OHDF group and the HD group. a pre-OHDF versus HD. $\mathbf{b}$ High-volume pre-OHDF (median) versus HD. c Low-volume pre-OHDF (median) versus $H D$. $H D$, hemodialysis; $H R$, hazard ratio; OHDF, online hemodiafiltration; pre-OHDF, predilution online hemodiafiltration; post-OHDF, post-dilution online hemodiafiltration; HR, hazard ratio; $\mathrm{Cl}$, confidence interval

divided by the median or weighted average of the substitution volume (Additional file 12: Figure S2) and of the CV corrected body surface area $(P=0.980$ and $P=0.540$, log-rank test).

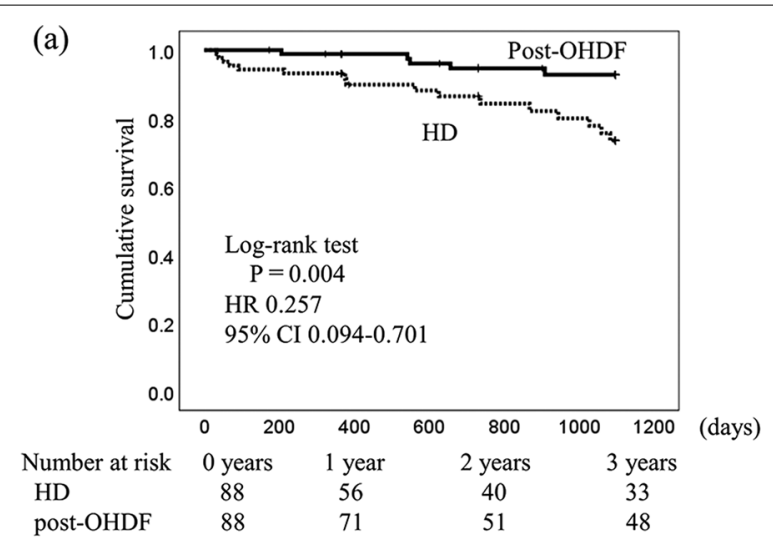

(b)

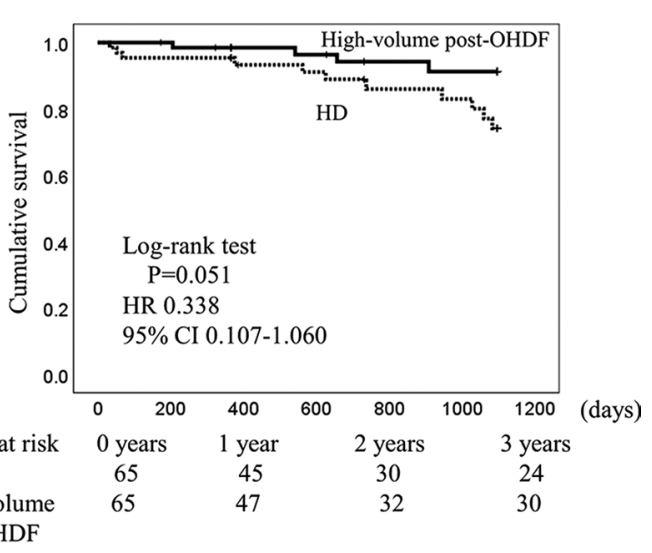

(c)

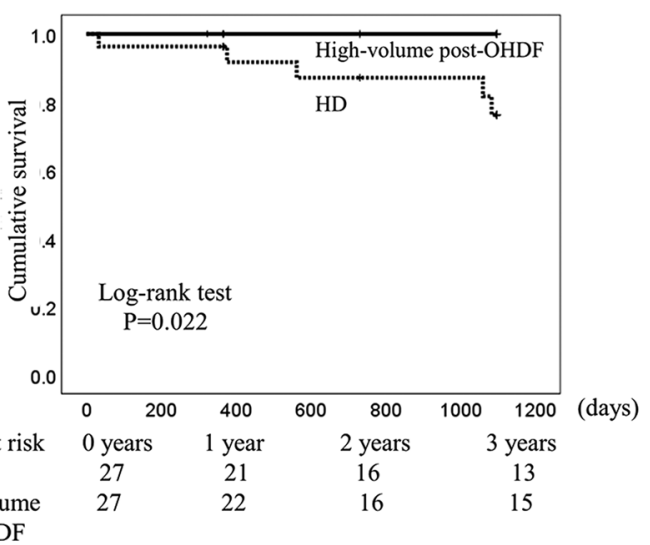

post-OHDF

Fig. 3 Comparison of patient survival outcomes between the post-OHDF group and the HD group. a Post-OHDF (median) versus HD. b High-volume post-OHDF (median) versus HD. c High-volume post-OHDF (weighted average) versus HD. HD, hemodialysis; post-OHDF, post-dilution online hemodiafiltration. HR, hazard ratio; $\mathrm{Cl}$, confidence interval
Comparison of total annual number of hospitalizations and cardiovascular events in the OHDF, pre-OHDF, and post-OHDF groups versus the HD group The OHDF group had a significantly lower number 


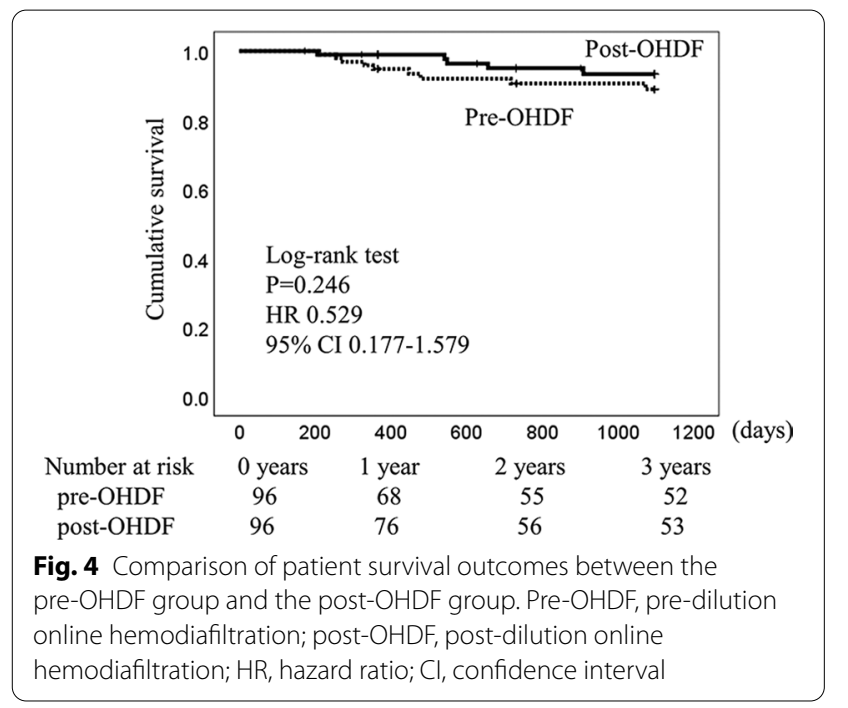

of hospitalizations and cardiovascular events in some years, and the post-OHDF group in particular had a significantly lower number of cardiovascular events in all 3 years compared with the HD group (Table 3).

\section{Discussion}

Our corporation, which includes one hospital and six clinics, has created a unified basic policy for healthcare management with regard to dialysis conditions, dry weight, chronic kidney disease-mineral and bone disorders, renal anemia, and vascular access to eliminate any differences in healthcare quality at each facility. Furthermore, the Blood Purification Management Center regularly controls blood pressure levels before dialysis, the cardiothoracic ratio, fluid replacement times for dialysis-induced hypotension, serum albumin, phosphorus, corrected calcium, intact-parathyroid hormone,

Table 2 Results of PSM in the high-volume versus low-volume pre-OHDF groups divided by the median

\begin{tabular}{|c|c|c|c|c|c|c|}
\hline \multirow[t]{2}{*}{ Item } & \multicolumn{3}{|c|}{ Before matching } & \multicolumn{3}{|c|}{ After matching } \\
\hline & Low volume & High volume & $P$-value & Low volume & High volume & $P$-value \\
\hline Total $(n)$ & 144 & 201 & & 144 & 144 & \\
\hline Sex, male/female (\%) & $61.8 / 38.2$ & $69.2 / 30.8$ & 0.167 & $38.8 / 31.2$ & $67.4 / 32.6$ & 0.388 \\
\hline Underlying disease & & & 0.429 & & & 0.700 \\
\hline Chronic nephropathy (\%) & 34.7 & 35.8 & & 37.5 & 36.1 & \\
\hline Diabetic nephropathy (\%) & 32.5 & 30.8 & & 34.7 & 31.9 & \\
\hline Nephrosclerosis (\%) & 9.7 & 8.0 & & 9.7 & 8.3 & \\
\hline Other (\%) & 18.1 & 25.4 & & 18.1 & 23.6 & \\
\hline Diabetes mellitus, yes/no (\%) & $37.5 / 62.5$ & $32.3 / 62.7$ & 0.359 & $37.5 / 62.5$ & $33.3 / 66.7$ & 0.538 \\
\hline History of myocardial infarction, yes/no (\%) & 1.4/98.6 & $2.5 / 97.5$ & 0.704 & 1.4/98.6 & $2.8 / 97.2$ & 0.684 \\
\hline History of intracerebral bleeding, yes/no (\%) & $0.7 / 99.3$ & 1.0/99.0 & $>0.999$ & $0.7 / 99.3$ & $0.7 / 99.3$ & $>0.999$ \\
\hline History of cerebral infarction, yes/no (\%) & $4.2 / 95.8$ & $2.5 / 97.5$ & 0.536 & $4.2 / 95.8$ & $2.8 / 97.2$ & 0.750 \\
\hline History of quadruple amputation, yes/no (\%) & $2.1 / 97.9$ & $0.5 / 99.5$ & 0.312 & $2.1 / 97.9$ & $0.7 / 99.3$ & 0.622 \\
\hline Use of antihypertensive agents, yes/no (\%) & $37.5 / 62.5$ & $37.3 / 62.7$ & $>0.999$ & $37.5 / 62.5$ & $38.9 / 61.4$ & 0.904 \\
\hline Age (years) & $66.1 \pm 11.7$ & $64.9 \pm 11.8$ & 0.316 & $66.1 \pm 11.7$ & $65.1 \pm 10.9$ & 0.266 \\
\hline Dialysis vintage (months) & $139.8 \pm 124.2$ & $148.4 \pm 114.7$ & 0.212 & $139.8 \pm 124.2$ & $157.8 \pm 116.0$ & 0.052 \\
\hline Blood flow rate (mL/min) & $277.9 \pm 18.9$ & $179.7 \pm 20.2$ & 0.155 & $270.0 \pm 19.3$ & $176.6 \pm 19.7$ & 0.136 \\
\hline Duration of dialysis session (h) & $4 \pm 0$ & $4 \pm 0$ & $>0.999$ & $4 \pm 0$ & $4 \pm 0$ & $>0.999$ \\
\hline Systolic BP, predialysis (mmHg) & $141 \pm 25$ & $145 \pm 27$ & 0.354 & $141 \pm 25$ & $143 \pm 27$ & 0.650 \\
\hline Diastolic BP, predialysis (mmHg) & $75 \pm 14$ & $78 \pm 15$ & 0.096 & $75 \pm 14$ & $77 \pm 14$ & 0.473 \\
\hline Body mass index (calculated as kg/m²) & $22.6 \pm 3.9$ & $22.8 \pm 3.7$ & 0.353 & $22.6 \pm 3.9$ & $22.7 \pm 3.7$ & 0.622 \\
\hline Albumin, predialysis (g/dL) & $3.4 \pm 0.3$ & $3.4 \pm 0.3$ & 0.681 & $3.4 \pm 0.3$ & $3.4 \pm 0.3$ & 0.910 \\
\hline High-sensitivity CRP, predialysis (g/dL) & $0.403 \pm 1.109$ & $0.403 \pm 1.134$ & 0.265 & $0.403 \pm 1.109$ & $0.360 \pm 1.098$ & 0.103 \\
\hline Corrected calcium, predialysis (m/dL) & $9.2 \pm 0.7$ & $9.2 \pm 0.7$ & 0.354 & $9.2 \pm 0.7$ & $9.2 \pm 0.7$ & 0.500 \\
\hline Phosphorus, predialysis (m/dL) & $5.6 \pm 1.5$ & $5.5 \pm 1.3$ & 0.768 & $5.6 \pm 1.5$ & $5.6 \pm 1.3$ & 0.787 \\
\hline Hemoglobin, predialysis (m/dL) & $11.2 \pm 1.4$ & $11.1 \pm 1.2$ & 0.324 & $11.2 \pm 1.4$ & $11.0 \pm 1.2$ & 0.128 \\
\hline Ferritin $(\mathrm{ng} / \mathrm{mL})$ & $68.1 \pm 77.1$ & $81.2 \pm 181.8$ & 0.462 & $76.6 \pm 96.3$ & $75.3 \pm 157.7$ & 0.650 \\
\hline $\mathrm{Kt} / \mathrm{N}$ & $1.6 \pm 0.3$ & $1.5 \pm 0.3$ & $<0.05$ & $1.6 \pm 0.3$ & $1.5 \pm 0.3$ & 0.069 \\
\hline Creatinine, predialysis (mg/dL) & $10.86 \pm 2.95$ & $11.46 \pm 2.78$ & $<0.05$ & $10.86 \pm 2.96$ & $11.29 \pm 2.80$ & 0.143 \\
\hline Blood urea nitrogen, predialysis (mg/dL) & $58.5 \pm 15.3$ & $59.4 \pm 14.3$ & 0.303 & $58.5 \pm 15.3$ & $59.4 \pm 13.8$ & 0.334 \\
\hline
\end{tabular}

PSM, propensity score matching; pre-OHDF, pre-dilution online hemodiafiltration; BP, blood pressure; CRP, C-reactive protein 


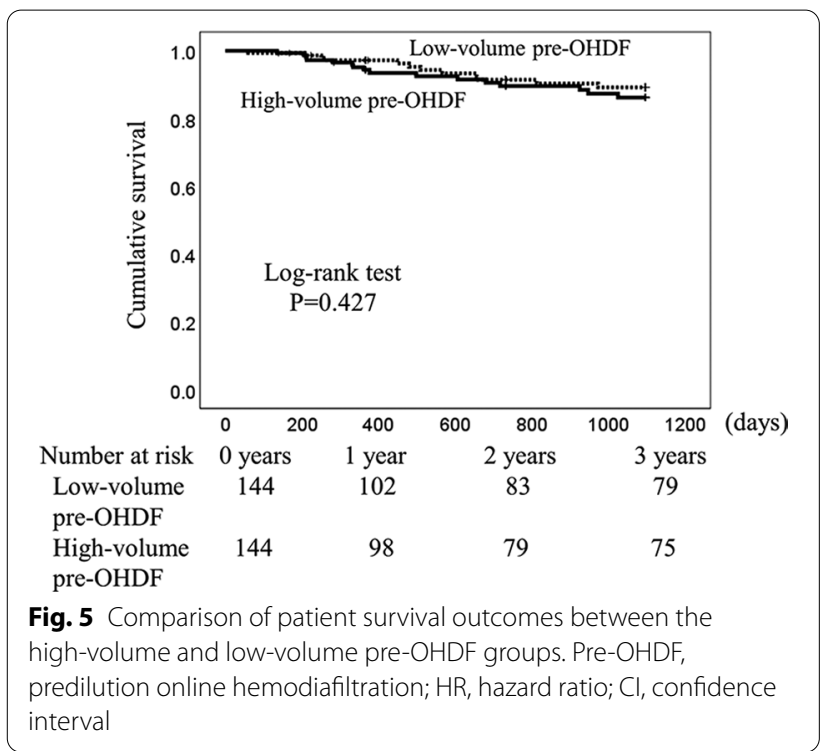

hemoglobin, ferritin, and transferrin saturation, $\mathrm{Kt} / \mathrm{V}$, and $\beta_{2}$ MG values. The center checks that the control status is within the standard range. The QB level is $250-300 \mathrm{~mL} / \mathrm{min}$ in principle, and the total QD level is $500 \mathrm{~mL} / \mathrm{min}$. There seems to be no significant difference in unobserved background factors, such as quality of healthcare management and dialysis conditions. Because the average QB at our facilities is approximately $280 \mathrm{~mL} /$ min, both pre-OHDF and post-OHDF are options. If patients have symptoms such as pruritus, restless legs syndrome, fatigue, or osteoarticular pain, pre-OHDF or post-OHDF is selected at the physician's discretion. If the patient has dialysis hypotension or cramps during dialysis, pre-OHDF is often selected because it is expected to improve them. We select the hemodiafilter based on the serum albumin level. Then, we determine the substitution volume based on the albumin leakage of the selected hemodiafilter. Switching from pre-OHDF to post-OHDF is considered when $\mathrm{Kt} / \mathrm{V}$ falls below 1.4 during preOHDF. If symptoms do not improve, we increase the substitution volume, change the hemodiafilter, or change the dilution method, depending on the serum albumin level. Additionally, in our experience, aggressive albumin leakage according to the serum albumin level is important because many patients have improvement in symptoms with increasing albumin leakage.

A meta-analysis of six randomized controlled trials (RCTs) indicated that all-cause mortality was significantly lower in post-OHDF than in HD and that the risk of mortality was lower in post-OHDF with a CV greater than $20 \mathrm{~L}$ per session [4]. Another meta-analysis of four RCTs demonstrated that all-cause mortality was reduced when $C V$ was higher, regardless of whether it was standardized to body surface area and total body water [5]. On the other hand, two prospective randomized studies [6, 7] and a prospective observational study of substitution volume $>20 \mathrm{~L}$ [8] failed to demonstrate a survival advantage of post-OHDF over HD.

In recent years, data from the JRDR database have demonstrated that all-cause mortality is improved by pre-OHDF compared with HD and that the optimal substitution volume is $50.5 \mathrm{~L}$ with a range of 39.0-63.5 L [2]. However, the JRDR database contains little data on QD and total QD and cannot capture differences in the quality of healthcare management between institutions, all of which affect prognosis. It should be noted that differences in unobserved background factors (quality of healthcare management and some dialysis conditions) cannot be adjusted for even if all observed background factors are adjusted by PSM. Moreover, there has been no report on the clinical effects of Japanese-style post-OHDF.

Recently, in the context of evidence-based medicine, focus has been placed on the reliability of studies requiring a large number of patients without significant differences in observed background factors at baseline, as in the JRDR database. However, the JRDR database cannot evaluate the quality of healthcare management or QD or total QD [2]. Therefore, we believe that our results using PSM with caliper values [3] would not be inferior to the evidence from the JRDR database even if there were significant differences in observed background factors.

Table 3 Comparison of annual hospitalizations and cardiovascular events in OHDF, pre-OHDF, and post-OHDF groups versus the HD group

\begin{tabular}{llllllll}
\hline Comparison group & \multicolumn{2}{l}{ Annual hospitalizations } & & \multicolumn{3}{l}{ Annual cardiovascular events } \\
\cline { 2 - 3 } & $\mathbf{2 0 1 7 - 2 0 1 8}$ & $\mathbf{2 0 1 8 - 2 0 1 9}$ & $\mathbf{2 0 1 9 - 2 0 2 0}$ & & $\mathbf{2 0 1 7 - 2 0 1 8}$ & $\mathbf{2 0 1 8 - 2 0 1 9}$ & $\mathbf{2 0 1 9 - 2 0 2 0}$ \\
\hline HD versus OHDF & $P<0.05$ & $P<0.05$ & $P=0.685$ & & $P=0.566$ & $P<0.05$ & $P=0.102$ \\
HD versus pre-OHDF & $P=0.291$ & $P=0.193$ & $P=0.906$ & & $P=0.083$ & $P=0.083$ & $P=0.126$ \\
HD versus post-OHDF & $P<0.05$ & $P=0.119$ & $P=0.558$ & & $P<0.05$ & $P<0.01$ & $P<0.01$ \\
\hline
\end{tabular}

$\mathrm{HD}$, hemodialysis; OHDF, online hemodiafiltration; post-OHDF, post-dilution online hemodiafiltration; pre-OHDF, pre-dilution online hemodiafiltration

The $p$-value was calculated using the Mann-Whitney $\mathrm{U}$ test 
Indeed, the observed background factors in the OHDF, pre-OHDF, and post-OHDF groups were significantly different after PSM using reliable caliper values. However, there was a significant improvement in all-cause mortality in the OHDF group, which contained a relatively large number of patients in this study, even though significant differences in background factors disappeared when the caliper value was lowered.

Three-year all-cause mortality was significantly lower in the OHDF, pre-OHDF, and post-OHDF groups compared with the HD group. This study is the first to show that Japanese-style post-OHDF as well as European-style post-OHDF [1] improves survival by reduction of cardiovascular events to a greater degree than HD. Mortality was not significantly different between the low-volume post-OHDF group and the HD group but was significantly lower in the high-volume post-OHDF group compared with the HD group. The ESHOL study showed that post-OHDF with a mean substitution volume of $21.8 \mathrm{~L}$ had a $33 \%$ lower risk $(P=0.06)$ of cardiovascular mortality and a Turkish study found that mortality was significantly lower with post-OHDF with a median substitution volume greater than $17.4 \mathrm{~L}$ compared with $\mathrm{HD}[1,7]$.

In an earlier JRDR database study, the mean substitution volumes in pre-OHDF and post-OHDF were 39.9 $\mathrm{L}$ and $10.2 \mathrm{~L}$, respectively [9]. Our finding regarding the effect of pre-OHDF substitution volume on survival is different from that in a previous dose-response study [2] because the substitution volume in pre-OHDF was $\geq 60 \mathrm{~L}$ in $97.1 \%$ of cases.

This paper suggests that survival outcomes are better because of decreased cardiovascular events in patients who receive Japanese-style post-OHDF, particularly with a substitution volume over $12 \mathrm{~L}$, compared with those who receive HD, that pre-OHDF with a substitution volume of 72-84 L can similarly improve survival as compared with a substitution volume of $60 \mathrm{~L}$, and that pre-OHDF and post-OHDF can also improve survival to the same degree. The major limitation of this study was the significant differences in some observed background factors after PSM, which can be attributed to the small number of cases. We do not have data on residual kidney function, although the dialysis vintage for patients receiving HD or OHDF was more than 3 months. An RCT is needed in the future to confirm our findings.

\section{Conclusions}

This study is the first to suggest that Japanese-style post-OHDF, particularly high-volume post-OHDF, may improve all-cause mortality by suppressing cardiovascular events, and high-volume of $72 \mathrm{~L}$ to $84 \mathrm{~L}$ pre-OHDF may not worsen all-cause mortality.

\section{Abbreviations}

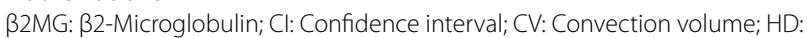
Hemodialysis; HR: Hazard ratio; OHDF: Online hemodiafiltration; post-OHDF: Post-dilution online hemodiafiltration; pre-OHDF: Predilution online hemodiafiltration; PSM: Propensity score matching; QB: Blood flow rate; QD: Dialysate flow rate; RCT: Randomized controlled trial.

\section{Supplementary Information}

The online version contains supplementary material available at https://doi. org/10.1186/s41100-021-00385-1.

Additional file 1: Table S1. Comparison of variables before and after propensity score matching in the hemodialysis (HD) vs. online hemodiafiltration (OHDF) groups.

Additional file 2: Table S2. Comparison of variables before and after propensity score matching in the hemodialysis (HD) vs. predilution online hemodiafiltration (pre-OHDF) groups.

Additional file 3: Table S3. Comparison of variables before and after propensity score matching in the hemodialysis (HD) vs. postdilution online hemodiafiltration (post-OHDF) groups.

Additional file 4: Table S4. Comparison of variables before and after propensity score matching in the hemodialysis (HD) vs. high-volume predilution online hemodiafiltration (pre-OHDF) groups divided by the median.

Additional file 5: Table S5. Comparison of variables before and after propensity score matching in the hemodialysis (HD) vs. low-volume predilution online hemodiafiltration (pre-OHDF) groups divided by the median

Additional file 6: Table S6. Comparison of variables before and after propensity score matching in the hemodialysis (HD) vs. high-volume postdilution online hemodiafiltration (post-OHDF) groups divided by the median

Additional file 7: Table S7. Comparison of the variables before and after propensity score matching in the hemodialysis (HD) vs. high-volume postdilution online hemodiafiltration (post-OHDF) groups divided by the weighted average.

Additional file 8: Table S8. Comparison of variables before and after propensity score matching in the hemodialysis (HD) vs. low-volume postdilution online hemodiafiltration (post-OHDF) groups divided by the median.

Additional file 9: Table 59 Comparison of variables before and after propensity score matching in the hemodialysis (HD) vs. low-volume postdilution online hemodiafiltration (post-OHDF) groups divided by the weighted average

Additional file 10: Table S10. Comparison of variables before and after propensity score matching in the predilution online hemodiafiltration (pre-OHDF) vs. postdilution online hemodiafiltration (post-OHDF) groups.

Additional file 11: Figure S1. Comparison of patient survival outcomes for the OHDF groups vs. the HD group.

Additional file 12: Figure S2. Comparison of patient survival outcomes for high-volume vs. low-volume post-OHDF groups (a) High-volume vs. lowvolume post-OHDF (median) (b) High-volume vs. low-volume post-OHDF (weighted average)

\section{Acknowledgements}

We are grateful to all the staff in our medical corporation for providing a consistent quality of healthcare management and dialysis conditions. We are also grateful to Dr. Shigeaki Ohtsuki of Japan Institute of Statistical Technology for the statistical analysis.

\section{Authors' contributions}

$\mathrm{KO}$ interpreted the results of statistical analysis and was the major contributor to drafting the manuscript, and HM, MT, TI, HS, JM, and SK performed data collection. All authors read and approved the final manuscript. 


\section{Funding}

This research received a specific grant from Nipro Co., Ltd for the statistical analysis. Nipro Co., Ltd was involved in the study design and had no role in the collection, analysis, or interpretation of data, in the writing of the report, or in the decision to submit the article for publication.

\section{Availability of data and materials}

All data generated or analyzed during this study are included in this published article and its supplementary information files.

\section{Declarations}

\section{Ethics approval and consent to participate}

This study was approved by the Research Ethics Committee of Kawashima Hospital and all clinical investigations were conducted according to the principles expressed in the Declaration of Helsinki. All patients gave informed consent for their data to be included in the study.

\section{Consent for publication}

Not applicable.

\section{Competing interests}

All the authors report the funding of specific clinical research (Japan Registry of Clinical Trials registration number jRCTs062190020) from Asahi Kasei Medical Co., Ltd. and contract research from Nipro Co., Ltd.

\section{Author details}

${ }^{1}$ Department of Nephrology, Kawashima Hospital, 6-1 Kitasakoichiban-cho, Tokushima-shi, Tokushima 770-0011, Japan. ${ }^{2}$ Department of Clinical Engineering, Kawashima Hospital, Tokushima, Japan.

Received: 16 September 2021 Accepted: 29 November 2021

Published online: 23 December 2021

\section{References}

1. Maduell F, Moreso F, Pons M, Ramos R, Mora-Macià J, Carreras J, et al. High-efficiency postdilution online hemodiafiltration reduces all-cause mortality in hemodialysis patients. J Am Soc Nephrol. 2013;24:487-97.

2. Kikuchi K, Hamano T, Wada A, Nakai S, Masakane I. Predilution online hemodiafiltration is associated with improved survival compared with hemodialysis. Kidney Int. 2019;95:929-38.

3. Austin PC. Optimal caliper widths for propensity-score matching when estimating differences in means and differences in proportions in observational studies. Pharmaceut Statist. 2011;10:150-61.

4. Mostovaya IM, Blankestijn PJ, Bots ML, Covic A, Davenport A, Grooteman MPC, et al. Clinical evidence on hemodiafiltration: a systematic review and a meta-analysis. Semin Dial. 2014;27:119-27.

5. Davenport A, Peters SAE, Bots ML, Canaud B, Grooteman MPC, Asci G, et al. Higher convection volume exchange with online hemodiafiltration is associated with survival advantage for dialysis patients: the effect of adjustment for body size. Kidney Int. 2016;89:193-9.

6. Grooteman MPC, van den Dorpel MA, Bots ML, Penne $L$, van der Weerd NC, Mazairac AHA, at al. Effect of online hemodiafiltration on all-cause mortality and cardiovascular outcomes. J Am Soc Nephrol. 2012:23:1087-96.

7. Ok E, Asci G, Toz H, Ok ES, Kircelli F, Yilmaz M, et al. Mortality and cardiovascular events in online haemodiafiltration (OL-HDF) compared with high-flux dialysis: results from the Turkish OL-HDF Study. Nephrol Dial Transpl. 2013;28:192-202.

8. Locatelli F, Karaboyas A, Pisoni RL, Robinson BM, Fort J, Vanholder R, et al. Mortality risk in patients on hemodiafiltration versus hemodialysis: a'realworld' comparison from the DOPPS. Nephrol Dial Transpl. 2018;33:683-9.

9. Masakane I, Taniguchi M, Nakai S, Tsuchida K, Wada A, Ogata S, et al. Annual dialysis data report 2016, JSDT renal data registry. Ren Replace Ther. 2018;4:45.

\section{Publisher's Note}

Springer Nature remains neutral with regard to jurisdictional claims in published maps and institutional affiliations.
Ready to submit your research? Choose BMC and benefit from:

- fast, convenient online submission

- thorough peer review by experienced researchers in your field

- rapid publication on acceptance

- support for research data, including large and complex data types

- gold Open Access which fosters wider collaboration and increased citations

- maximum visibility for your research: over 100M website views per year

At $\mathrm{BMC}$, research is always in progress.

Learn more biomedcentral.com/submissions 